\title{
Cas9/gRNA-mediated modifications of the barley genome for fundamental and applied research
}

Gerasimova S. ${ }^{1,2}$, Egorova A. ${ }^{1,2,3}$, Hertig C. ${ }^{3}$, Korotkova A. ${ }^{1}$, Kolosovskaya E. ${ }^{2}$, Strygina K. ${ }^{4}$, Shoeva O. ${ }^{1}$, Koeppel I. ${ }^{3}$, Hiekel S. ${ }^{3}$, Vikhorev A. ${ }^{2}$, Shmakov N. ${ }^{1}$, Kochetov A. ${ }^{1}$, Kumlehn J. ${ }^{3}$, Khlestkina E.K. ${ }^{4}$

${ }^{1}$ Kurchatov Genomic Center of the Institute of Cytology and Genetics, SB RAS, Novosibirsk, Russia

${ }^{2}$ Novosibirsk State University, Novosibirsk, Russia

${ }^{3}$ Leibniz Institute of Plant Genetics and Crop Plant Research, Gatersleben, Germany

${ }^{4}$ N.I. Vavilov All-Russian Institute of Plant Genetic Resources, St. Petersburg, Russia

*email: gerson@bionet.nsc.ru

Methods of targeted genome modification provide great opportunities for reverse genetics, the generation of experimental models and trait improvement in crop plants. All these strategies have been pursued in barley research in close cooperation between the Institute of Cytology and Genetics (ICG Novosibirsk) and the Leibniz Institute of Plant Genetics and Crop Plant Research (IPK Gatersleben). The comparatively straightforward Cas9/gRNA technology allows one to knockout virtually any gene of interest. As a first concerted effort using this endonuclease platform, the NUDUM $(N U D)$ gene was knocked out in order to convert hulled into naked barley. Lines with homozygous mutations in this gene exhibit the naked grain phenotype (i.e. with nonadherent hull) and do not differ from the wild-type in any other agronomically relevant characteristics. Naked barley is an exceedingly valuable dietary component, and the exemplified principle of phenotype conversion is currently being applied for elite Siberian barley germplasm. In another line of research, several genes controlling barley grain color have been identified. To confirm their anticipated functions, barley plants carrying mutations in some of these genes were generated. As was envisaged in the experimental concepts, the obtained mutations comprised both loss-of-function and gain-of-function modifications. To establish new experimental models for basic research, some barley lines carrying homozygous mutations in unknown or poorly studied APETALA2/ETHYLENE-RESPONSIVE FACTOR (AP2/ERF) transcription factor family genes were developed. The loss-of-function of one of these genes led to a glossy leaf sheath phenotype with epicuticular wax deficiency. A comprehensive investigation of such mutants allowed us to elucidate a hitherto unknown control mechanism of epicuticular wax accumulation in barley. Taken together, the obtained results already provide an idea of the unprecedented power of Cas9/gRNA technology in crop research, the implementation of which is still in its infancy.

Acknowledgements: The study is supported by the RSF (21-66-00012) and by the Kurchatov Genomic Center of the Institute of Cytology and Genetics, SB RAS (075-152019-1662). 